\title{
Le stress au travail : du paradigme des Risques Psychosociaux à celui de la Qualité de Vie au Travail
}

\section{Occupational stress: from the paradigm of Psychosocial Risks to that of Quality of Work Life}

\section{Céline Sauvezon*}

Univ Paul Valéry Montpellier 3, Univ. Montpellier, EPSYLON EA 4556, F34000, Montpellier, France

\section{Dominique Ferrieux}

Univ Paul Valéry Montpellier 3, Univ. Montpellier, EPSYLON EA 4556, F34000, Montpellier, France

\section{Daniel Priolo}

Univ Paul Valéry Montpellier 3, Univ. Montpellier, EPSYLON EA 4556, F34000, Montpellier, France

\section{* Auteur correspondant}

Univ. Paul Valéry Montpellier 3, Univ. Montpellier, EPSYLON EA 4556, F34000, Montpellier, France

04.67.14.20.00

celine.sauvezon@univ-montp3.fr 


\title{
Résumé
}

Une recherche-action utilisant trois modèles classiques du stress au travail nous a permis de vérifier l'impact sur les résultats obtenus du choix du modèle et du paradigme: Risques Psychosociaux versus Qualité de Vie au Travail. Quatre cent trente-six salariés de la grande distribution ont répondu à un questionnaire incluant ces trois mesures du stress, les conséquences (satisfaction, fatigue) et une cause possible (tensions de rôle). Nos résultats valident les hypothèses de différences significatives des niveaux de prévalence du stress selon le modèle, comme des liens avec le facteur et les conséquences, ainsi que l'importance de la perception de stress pour expliquer le vécu négatif.

Mots clé : stress au travail, risque psychosociaux, qualité de vie au travail, tension de rôle, satisfaction au travail

\begin{abstract}
An action research using three classical models of occupational stress enabled us to verify the impact on the results obtained from the choice of model and paradigm: Psychosocial Risks versus Quality of Work Life. Four hundred and thirty-six supermarket employees answered a questionnaire including these three measures of stress, the outcomes (satisfaction, fatigue) and a possible cause (role strain). Our results validate the hypotheses of significant differences in stress prevalence levels according to the model, such as links to the factor and the consequences, as well as the importance of the perception of stress to explain the negative experience.
\end{abstract}

Key words: occupational stress, psychosocial risks, quality of work life, role strain, job satisfaction

Pas de conflits d'intérêts 


\section{Introduction}

L'article s'inscrit dans le cadre d'une recherche-action sur la santé psychologique au travail dans une entreprise de la grande distribution du sud de la France. Il s'agissait d'accompagner les acteurs de l'entreprise dans un diagnostic du stress au travail et la recherche de solutions.

Le premier objectif de l'article est de montrer l'intérêt d'un recul réflexif par rapport aux choix méthodologiques et théoriques nécessaires pour toute intervention en entreprise sur le stress au travail. Plusieurs modèles théoriques classiques existent, internationalement connus et utilisés : e.g. Karasek et Theorell, 1990 ; Siegrist, 1991 ; Lazarus et Folkman, 1984. Toutefois, la force et la nature des liens qu'entretiennent ces modèles avec d'autres variables (facteurs ou conséquences du stress) et donc les conséquences du choix de l'un ou l'autre sur la compréhension des phénomènes et les préconisations qui en découleront ne sont pas forcément explicités. Ce sont ces différences dans les résultats obtenus lors du diagnostic et leur impact sur l'évaluation de l'origine et du niveau de stress, donc sur les solutions recherchées, que nous voulions préciser.

Par ailleurs, sur le plan plus fondamental de la recherche, deux paradigmes coexistent dans les travaux sur la santé au travail : celui des Risques Psychosociaux (RPS) et celui de la Qualité de vie au Travail (QVT). Le deuxième objectif vise à souligner l'intérêt d'un changement de paradigme pour développer une vision plus équilibrée et synthétique de la santé psychologique au travail (SPT), trop longtemps limitée à la mise en évidence des dimensions négatives du travail à travers une approche épidémiologique.

\section{Cadre conceptuel et théorique : le stress au travail}

Une toute première conception béhavioriste et physiologique (Selye, 1956) du stress a rapidement évolué vers une conception interactionniste où «c'est la conjonction entre certaines caractéristiques de l'environnement et/ou de la personne qui constitue un «facteur de risque » (Rascle \& Irachabal, 2001, p. 101).

Le modèle Job Demand-Control-Support (JDC-S) de Karasek et Theorell (1990) s'inscrit dans cette approche. Le stress au travail résulterait de l'interaction de 3 dimensions de l'environnement de travail : la demande psychologique (exigences du travail en termes de quantité, rapidité, complexité...), la latitude décisionnelle (autonomie et possibilité d'utiliser 
et développer ses compétences) et le soutien social (émotionnel et technique) venant des collègues et des supérieurs hiérarchiques.

Une situation de travail croisant une forte demande psychologique et une faible latitude décisionnelle entraîne du jobstrain et de l'isostrain si en plus le soutien social est faible. L'outil qui accompagne ce modèle, le Job Content Questionary (JCQ), est une référence mondiale de mesure du stress au travail (Van Wassenhove, 2014). Ce modèle, validé par de nombreuses études scientifiques, établit les liens entre jobstrain, isostrain et divers problèmes liés au travail ou des pathologies physiques ou mentales.

Cependant, les 3 dimensions combinatoires sont parfois considérées plutôt comme des dimensions indépendantes ayant des effets différenciés (Le Blanc, de Jonge \& Schaufeli, 2000; Guillet, Hermand, \& Py, 2003). Par ailleurs, le modèle ne rend pas compte de toutes les situations de stress au travail. Niedhammer, Chastan, Gendrey, David et Degioanni, (2006) montrent que la prévalence du jobstrain+isostrain ne représente que $40.45 \%$ des situations de travail perçues comme fortement stressantes chez les hommes. On peut trouver plusieurs raisons à cela : le modèle repose sur l'analyse d'un petit nombre de facteurs dont les liens avec le stress sont déduits sur la base des corrélations constatées avec des problèmes de santé, sans mesurer si le sujet perçoit subjectivement ces situations comme stressantes ni la façon spécifique dont il va s'y adapter. Le sujet est ici passif face à une situation qui représente épidémiologiquement un risque pour sa santé : le travail n'est qu'un facteur de risque, pas une source de créativité où l'acteur construit sa santé en donnant du sens et influençant son environnement (Clot, 2015).

Dans le modèle Effort-Reward Imbalance (ERI) de SIEGRIST (1991), le stress résulte d'une perception par le sujet d'un déséquilibre dans sa situation de travail entre ses efforts extrinsèques (contraintes de temps, responsabilités...) et les récompenses reçues en retour (reconnaissance etc.). Le questionnaire évalue pour chaque facteur si sa présence ou son absence représente une source de tension pour le sujet, ce qui explique que certains auteurs classent ce modèle parmi les modèles transactionnels. Néanmoins, comme dans le JDC-S le processus subjectif d'évaluation du sens que le sujet attribue spécifiquement à la situation n'est pas pris en compte.

La troisième conception, transactionnelle, va analyser les perceptions subjectives, les différences interindividuelles et les stratégies des acteurs dans le processus de stress. Pour Lazarus et Folkman (1984) «le stress est une relation particulière entre la personne et l'environnement, [...] évaluée par l'individu comme excédant ses ressources. » (p. 19). Il convient donc de distinguer «les caractéristiques objectives de l'environnement, ou 
stresseurs, des caractéristiques environnementales perçues comme menaçantes par un individu, ou stress perçu, d'une part, et des conséquences dysfonctionnelles éventuelles de ces facteurs ou ajustement ultérieur de cet individu, d'autre part » (Rascle \& al., 2001). Le stress résulte ici d'une double évaluation des enjeux de la situation (menace, défi ...) et des ressources et capacités d'actions perçues par le sujet. Une situation peut ainsi être évaluée comme plus ou moins stressante par le «salarié acteur » qui va lui accorder des significations spécifiques et adopter des stratégies d'ajustement différentes de celles d'autres acteurs. Le Perceveid Stress Scale (PSS) de Cohen, Tyrrell \& Smith (1993) peut être rattaché à ce modèle. Il estime le sentiment de débordement issu de la double évaluation et mesure ainsi la perception de stress vécue.

Ainsi selon l'approche, interactionniste ou transactionnelle, ces modèles définissent différemment le stress en lui-même: conséquence mécanique d'interaction de facteurs environnementaux ou processus subjectif d'évaluation de la situation. Ils définissent également différemment les facteurs du stress, tant dans leur nombre que dans leur nature.

D’autres approches ont été développées dans la littérature (e.g. psychologie cognitive, sociologie, clinique de l'activité, psychodynamique du travail). D'autres facteurs de stress au travail non inclus dans ces modèles existent, Gollac et Bodier (2011) en recensent une quarantaine dont les exigences émotionnelles, les tensions de rôles (Rizzo, House, \& Lirtzman, 1970), la justice organisationnelle (Elovainio et al., 2010), le style de management et les compétences managériales (Laberon, Barou, \& Ripon, 2016). Il paraît donc nécessaire d'ouvrir les travaux sur la SPT à la recherche d'un ensemble plus large de facteurs de stress. Toutefois, cet article ayant pour objectif de discuter de l'impact du choix du modèle sur les résultats obtenus et les interventions possibles, nous nous sommes volontairement centrés sur 3 des modèles les plus souvent mobilisés sur le terrain : JDC-S, ERI et PSS (Allard-Poesi \& Hollet-Haudebert, 2012).

Il parait également essentiel d'intégrer des mesures des conséquences de ce stress comme la satisfaction au travail ou la fatigue nerveuse (Ribert-Van de Weerdt, 2011) pour mieux comprendre comment l'effet des contraintes environnementales sur la SPT est médiatisé par la perception de stress et compléter ainsi l'approche épidémiologique, indispensable pour documenter les risques mais insuffisante pour comprendre le processus subjectif. 
Enfin, ces modèles définissent aussi différemment la place du sujet : «victime » passive des risques épidémiologiques que représente pour lui son travail (Allard-Poesi et al., 2012) ou «acteur» développant des comportements stratégiques visant à améliorer sa performance adaptative à la situation.

Ces écarts et limites trouvent un écho dans la façon dont deux paradigmes, RPS et QVT, nous proposent de concevoir la SPT.

Le paradigme RPS tend à considérer le travail comme un risque et à évaluer la santé au travail uniquement du point de vue des pathologies et des troubles de fonctionnement, d'où l'émergence de concepts tels que le stress ou la souffrance au travail (Dejours, 2000). La plupart des questionnaires de mesure de la santé psychologique sont ici composés de mesures d'anxiété et de dépression (Veit \& Ware, 1983). Cela pose un problème méthodologique. En se focalisant uniquement sur le risque que représente le travail pour la santé et sur des mesures exclusivement négatives de la SPT, on aboutit, d'une part à une surévaluation de la prévalence du stress (Savoie, Brunet, Boudrias, \& Gilbert 2010) et d'autre part on ignore en quoi le travail peut être un élément de construction de soi, de satisfaction, de créativité ... comme le proposent la psychologie positive et la clinique de l'activité (Clot, 2015).

Le paradigme QVT permet d'élargir l'analyse. La notion de QVT, apparue au début des années 1970 dans les pays anglo-saxons, renvoie aux conditions et aux caractéristiques du travail qui contribuent à la motivation, la performance et la satisfaction au travail. Elle reste une notion sans définition consensuelle, souvent associée à la SPT ou au bien-être au travail (Grosjean \& Guyot, 2016). Selon l'ANI (2013), la QVT vise d'abord le travail et ses conditions, et la possibilité de «faire du bon travail » dans une bonne ambiance. Ce paradigme s'inspire des travaux en psychologie positive (Seligman \& Csikszentmihalyi, 2000), davantage axés vers les aspects positifs de l'être humain.

Plutôt que chercher à seulement prévenir les risques et guérir les troubles, une approche QVT cherchera à identifier les variables pouvant favoriser l'efficacité organisationnelle et concevoir un travail qui prenne en compte les enjeux de santé. Elle propose donc un renversement de perspective avec une vision positive du travail (Grosjean \& Guyot, 2016).

La SPT peut alors être conçue comme un construit bi-dimensionnel, dans lequel chaque facteur de RPS peut aussi et simultanément être un élément de ressource (Burakova \& 
Leduc, 2014). Par exemple, une charge de travail élevée n'est pas forcément une cause de souffrance, elle peut être la marque d'un travail passionnant. A l'inverse une charge de travail trop faible, comme dans le cas extrême des salariés «placardisés », peut aussi être source de souffrances (Loriol, 2010). Le modèle de SPT de Gilbert, Dagenais-Desmarais et Savoie (2011) rejette aussi l'idée d'un continuum où la santé ne serait que l'absence de troubles. Ils proposent plutôt une conception bi-dimensionnelle de la SPT avec une mesure indépendante du bien-être et de la détresse psychologiques au travail.

Enfin, l'approche QVT identifie à la fois des facteurs subjectifs (bien être, satisfaction, ...) et structuraux (diversité du travail, participation, absentéisme, Burakova et al., 2014). La subjectivité de l'acteur et ses liens circulaires avec la performance et le contexte organisationnel ont ainsi plus d'espace pour être étudiés. Le salarié est alors considéré comme un acteur qui doit retrouver son «pouvoir d'agir», expert de ses propres contraintes, le seul à pouvoir dire si elles sont dans des limites raisonnables (Grosjean \& al, 2016).

\section{Objectifs et hypothèses}

Au vu de ces éléments théoriques se pose la question des conséquences du choix d'un modèle de stress au plan du diagnostic (sélection en amont des facteurs) puis de l'intervention (solutions vers lesquelles l'organisation et/ou l'intervenant seront orientés pour trouver des solutions d'amélioration). Notre hypothèse générale suppose que les résultats obtenus pour mesurer un même construit (le stress au travail) avec des modèles différents (JDC-S, ERI et PSS) seront différents. Autrement dit, la force des relations et la nature des effets (direct vs indirect) seront différents si nous utilisons le modèle ERI (Siegrist, 1991) plutôt que le modèle JDC-S (Karasek \& Theorell, 1990) ou le PSS (Cohen et al., 1993).

De par leur mode de calcul, les différentes prévalences du stress devraient se distinguer de manière significative en fonction du modèle utilisé (JDC-S vs. ERI vs. PSS). Nous faisons l'hypothèse que le JDC-S, basé sur la médiane, doit aboutir à une prévalence plus importante que l'ERI. Le PSS mesurant un processus transactionnel de stress dans les évènements de la vie à la fois travail et hors travail aboutira à une prévalence plus importante que les deux autres (H1).

Nous faisons ensuite l'hypothèse que les liens entre le stress et la SPT (satisfaction et fatigue nerveuse) n'auront pas la même intensité s'ils sont mesurés à l'aide de l'ERI plutôt qu'à l'aide du JDC-S ou du PSS (H2a). De plus, des écarts de même type se verront 
également dans les liens entre le stress et ses facteurs (tensions de rôle, H2b). La nature des relations (directe vs. indirecte) entre les tensions de rôle, le stress et la SPT peut également varier en fonction du modèle de stress choisi $(\mathrm{H} 2 \mathrm{c})$. Nous voulons ainsi souligner la nécessité d'intégrer des mesures des conséquences du stress aux modèles classiques qui se limitent à la perception de certaines dimensions de l'environnement ou à la subjectivité du processus d'évaluation de l'environnement. Nous voulons également montrer que selon le modèle choisi les conséquences du stress seront différentes, ce qui impacte le diagnostic et les préconisations.

Nous souhaitons également prendre en considération la bi-dimensionnalité de la SPT c'est-à-dire étudier les versants positifs comme négatifs de ce concept. Notre troisième hypothèse suppose qu'un facteur de stress (la tension de rôle) aura des effets tant sur le versant positif de la SPT (satisfaction, H3a) que sur le versant négatif (fatigue nerveuse, H3b).

Nous souhaitons enfin mettre en avant l'importance de la subjectivité des acteurs dans la compréhension des effets d'un facteur de stress sur la SPT. Pour bien comprendre les effets de la tension de rôle sur la SPT, la mesure du niveau de stress par des modèles interactionnistes (ERI ou JDC-S) est nécessaire mais pas suffisante. Ce type de stress (ERI ou JDC-S) peut avoir un effet sur la dimension subjective du stress (PSS). Nous faisons l'hypothèse que les effets d'un facteur de stress (tension de rôle) sur la SPT seront médiatisés par le niveau de stress mesuré à travers un modèle interactionniste (ERI ou JDC-S) et le stress perçu (PSS). Cette relation de médiation en piste causale constitue notre hypothèse 4. Elle s'observera sur les deux versants de la SPT : satisfaction au travail (H4a, figure 1) et fatigue nerveuse (H4b, figure 2).

INSERER FIGURE 1

INSERER FIGURE 2

\section{L'intervention :}

\section{a. Trois étapes}

Une première phase qualitative de 45 entretiens semi-directifs, a permis d'identifier les dimensions pertinentes du rapport au travail et à l'organisation. L'objectif était d'identifier la perception qu'avaient les acteurs de leur travail et de leur organisation comme par exemple les facteurs de stress évoqués et leurs conséquences mais aussi les ressources et les solutions. 
Les éléments recueillis ont permis de choisir les dimensions pertinentes à intégrer dans le questionnaire.

Une deuxième phase quantitative a évalué la prévalence de ces facteurs de risque et/ou de protection par rapport au stress au travail, ainsi que le niveau de gravité de ses conséquences, à partir d'un questionnaire proposé aux 1125 salariés de l'entreprise. Des échelles existantes ont été retenues, complétées avec des questions ad'hoc créées à partir de la pré-enquête lorsque nécessaire.

Ces évaluations ont débouché sur une troisième phase d'aide à l'élaboration de pistes d'action, au travers de l'animation de 5 groupes de travail constitués chacun de 12 salariés, représentatifs des différents services et statuts présents dans l'entreprise, qui ne sera pas discutée ici.

\section{b. Les outils}

Le questionnaire que nous avons adressé aux salariés était composé de plusieurs échelles mesurant de nombreux construits. Dans la présente recherche nous ne présenterons que ceux sur lesquels nous avons testé nos hypothèses. Ils peuvent être divisés en quatre catégories : les facteurs de stress au travail, les mesures interactionnistes de stress, la mesure transactionnelle de stress perçu et les mesures de conséquences du stress.

\section{Facteur de stress}

Nous avons choisi de présenter ici les résultats relatifs aux tensions de rôle (sentiment qu'éprouve une personne dans une situation où il lui est difficile, voire impossible, de répondre à toutes les attentes de façon satisfaisante tant à ses yeux qu'aux yeux des personnes qui les formulent, Katz et Kahn, 1978). Ce facteur comprend le conflit de rôle (le sujet est confronté à plus d'un rôle dont l'exercice de l'un rend difficile l'exercice de l'autre) et l'ambiguïté de rôle (incertitude par rapport aux comportements attendus). L'impact des tensions de rôles sur le stress a été démontré dans divers travaux (e.g., Xie, 2007 ; Ruiller, 2008 ; Codo, 2011) mais il reste, comparativement à d'autres variables, peu étudié en psychologie du travail et des organisations. Une échelle permettait de mesurer les tensions de rôle au travers de 9 items $(\alpha=.81)$ empruntés au questionnaire Sumer (2010) et à Rizzo et al. (1970) (e.g., Vous recevez parfois d'une ou plusieurs personnes des demandes incompatibles entre elles). L'échelle de réponse était en 4 points $(1=$ pas du tout d'accord ; 4 = tout à fait d'accord). 


\section{Mesures interactionnistes du stress}

Nous avons utilisé les 3 dimensions du JDC-S (Karasek et al., 1990) :

- la demande psychologique, 9 items $(\alpha=.88$, e.g., «mon travail me demande d'aller très vite »).

- La latitude décisionnelle, 9 items ( $\alpha=.86$, e.g., «j'ai la possibilité d'influencer le déroulement de mon travail $\gg)$.

- le soutien social, 8 items $(\alpha=.82$, e.g. «à quel point votre supérieur vous aide à mener à bien vos tâches »).

L'échelle de réponse était en 4 points (1 pas du tout ; 4 tout à fait).

Nous avons également utilisé l'ERI (Siegrist, 1991) pour évaluer les efforts extrinsèques (6 items, $\alpha=.68$ ) et les récompenses (11 items, $\alpha=.87$ ). Les participants devaient indiquer leur degré d'accord avec des items comme « $\mathrm{Vu}$ tous mes efforts, mon salaire est satisfaisant ». Nous avons calculé le ratio efforts/récompenses selon la formule de Siegrist (1991) après avoir, conformément à ses recommandations, recodé les échelles de Likert en 4 points ( 1 = pas du tout d'accord ; 4 = tout à fait d'accord $)$ sur une échelle en 2 points.

\section{Stress perçu}

Nous avons utilisé le PSS 10 de Cohen et al. (1993) pour évaluer le stress perçu. Pour chacun des 10 items $(\alpha=.85)$, les salariés devaient évaluer la fréquence d'événements sur une échelle en 5 points $(1=$ jamais ; 5 = souvent $)$, par exemple «Vous a-t-il semblé difficile de contrôler les choses importantes de votre vie ? ».

\section{Conséquences du stress}

Les conséquences du stress sur la SPT étaient appréhendées sous un versant positif (la satisfaction au travail) ainsi que sur un versant négatif (la fatigue nerveuse). Nous avons utilisé l'échelle de satisfaction au travail de Camman et al. (1983), composée de 3 items ( $\alpha=$ .85, e.g., «globalement je suis satisfait de mon travail»). L'état de fatigue nerveuse était évalué à l'aide d'une question issue de la phase de pré-enquête : «êtes-vous en ce moment nerveusement fatigué(e)? ». Pour ces deux mesures, les sujets devaient se positionner sur une échelle de Likert en 4 points ( 1 = pas du tout ; 4 = tout à fait).

\section{c. La population étudiée}


Nous avons envoyé par courrier une version papier du questionnaire au domicile de chaque salarié, avec une enveloppe adressée à une boîte postale pour le retour du questionnaire. La consigne précisait que seul les chercheurs auraient connaissance des réponses individuelles qui seraient confidentielles et anonymes. Nous avons reçu 436 questionnaires (38.8\% de la population), ce taux de réponse faible sera évoqué en conclusion. Cet échantillon présente des caractéristiques proches de la population des salariés de l'entreprise : $76.25 \%$ des répondants sont des hommes ( $80.44 \%$ de la population parente), $57.6 \%$ appartiennent à la CSP employés/ouvriers (60.71\% de la population parente), sauf pour le taux de réponse par type de service : les services logistiques représentent $48.2 \%$ de l'échantillon (60.17\% de la population). Nous pensons cependant disposer d'un échantillon de personnes suffisamment proche de la population des salariés de l'entreprise pour analyser les réponses aux différentes questions.

\section{Résultats}

\section{a. Prévalence du stress}

Insérer Tableau 1

Nous avons réalisé des $\chi^{2}$ yates pour comparer les prévalences des modèles deux à deux. Nous observons que la prévalence du stress diffère significativement entre le JDC-S (23.8\%) et l'ERI $(14.18 \%), \chi_{\text {yates }}^{2}(1)=12.51, p=.0004$. La différence de proportion de personnes stressées entre le JDC-S (23.8\%) et le PSS (42.33\%) est également significative, $\chi^{2}$ yates $(1)=$ 33.12, $p<.0001$, tout comme l'écart entre les estimations fondées sur l'ERI (14.18\%) et le PSS $(42.33 \%), \chi^{2}$ yates $(1)=84.07, p<.0001$. Tous ces résultats appuient notre hypothèse 1 . La prévalence du stress diffère selon le modèle de référence.

\section{b. Force du lien entre stress et santé psychologique au travail (SPT)}

Nous avons calculé des corrélations entre toutes les variables (voir tableau 2). Nous avons comparé l'intensité des valeurs absolues des corrélations entre les modèles (JDC-S vs ERI vs. PSS) et les deux aspects de la SPT (satisfaction au travail et fatigue nerveuse). Pour ce faire, nous avons procédé à un test d'homogénéité des corrélations qui utilisait les valeurs transformées de Fisher. Pour synthétiser ces comparaisons, nous pouvons dire que toutes les corrélations séparées de plus de 11 points sont statistiquement différentes. Ceci nous permet 
de mettre en évidence que l'ERI corrèle plus fortement que la latitude décisionnelle (JDC-S) avec la fatigue nerveuse (respectivement $r=.47 ; r=-.13, p<.0001$ ). Toutefois, le PSS semble être le modèle le plus indiqué pour comprendre la fatigue nerveuse $(r=.59)$. Par ailleurs, la latitude décisionnelle entretient des liens plus forts que la demande psychologique (JDC-S) et que le PSS avec la satisfaction au travail (respectivement $r=.48, r=-.31, r=-37$ ). Enfin, les tensions de rôles entretiennent des liens différents avec chacun des 3 modèles de stress. Les résultats indiquent que ces tensions sont plus fortement liées à l'ERI ( $r=.47)$, à la demande psychologique $(r=.48)$ et au soutien social $(r=-.54)$ qu'avec le PSS $(r=.36)$. Pris dans leur globalité ces résultats appuient nos hypothèses $2 \mathrm{a}$ et $2 \mathrm{~b}$. En effet, les relations entre les tensions de rôle, le stress au travail et la SPT (satisfaction et fatigue nerveuse) n'ont pas la même intensité en fonction du modèle de stress utilisé (JDC-S vs ERI vs. PSS).

Insérer tableau 2

\section{c. Tension de rôle, modèle de stress, stress perçu et} conséquences du stress

Pour tester nos hypothèses 2,3 , et 4 nous avons réalisé des tests de médiation utilisant la macro PROCESS développée par Hayes (2013). Nous avons effectué 10000 rééchantillonnages par bootstrap pour estimer l'erreur standard des effets indirects.

Afin de déterminer quelles dimensions du JDC-S retenir dans nos analyses de médiation, nous avons réalisé une modélisation en équation structurelle. Les résultats montrent que la latitude décisionnelle $(\beta=.30)$ était la plus adaptée pour comprendre les effets de la tension de rôle sur la satisfaction au travail. Pour la fatigue nerveuse c'était la demande psychologique $(\beta=.20)$ qui permettait la meilleure prédiction. Ces éléments sont conformes à d'autres travaux qui montrent l'importance de la latitude décisionnelle dans la satisfaction au travail (Karnas \& Hellemans, 2002) et/ou l'impact de la demande psychologique sur la fatigue (Dejours, 1993, 2000).

Tension de rôle TR (VI), ERI (VM1), PSS10 (VM2) et satisfaction au travail ST (VD)

Rappelons que conformément à notre hypothèse $3 \mathrm{a}$, plus la TR augmente plus la ST diminue $(r=-.51, p<.001)$. Concernant la médiation en série, l'ensemble des résultats sont présentés dans la figure 3 . Toutes les analyses nécessaires à cette médiation ont été réalisées. 
Par souci de concision, nous ne présenterons que les effets indirects. Puis nous les comparerons à l'aide de contrastes. Nous procèderons ainsi pour toutes les analyses de médiation.

Effet indirects $1 a, 2 a$ et $3 a$

Nous observons un effet indirect (indirect 1a) significatif de la TR sur la ST via l'ERI, $\beta=-.11, S E=.03,95 \%$ IC $=[-.16 ;-.06]$. Une partie de l'effet de la TR sur la ST passe uniquement par l'ERI.

Les résultats appuient l'hypothèse d'une médiation en série (indirect $2 \mathrm{a}$ ), $\beta=-.02, S E$ $=.01,95 \%$ IC $=[-.04 ;-.01]$. Autrement dit, la TR a un effet sur l'ERI qui a un effet sur le PSS qui a lui-même un effet sur la $\mathrm{ST}$, mais cet effet est très faible. Ce résultat est conforme à notre hypothèse $4 \mathrm{a}$.

Une partie de l'effet de la TR sur la ST est médiatisé uniquement par le PSS (indirect $3 a), \beta=-.04, S E=.02,95 \% I C=[-.07 ;-.01]$, mais cet effet est très faible.

\section{Comparaison des trois effets indirects}

L'indirect 1a (médiation simple par l'ERI) explique 23\% de l'effet total, l'indirect 2a (médiation en série) explique 4\% et l'indirect 3a (médiation simple par le PSS) explique 7\%. Les effets de la TR sur la ST sont principalement médiatisés par l'ERI. Ces résultats ne vont pas dans le sens de notre hypothèse $4 a$.

\section{INSERER FIGURE 3}

\section{Tension de rôle TR (TR - VI), latitude décisionnelle LD (VM1), PSS (VM2) et satisfaction au travail ST (VD)}

Les résultats concernant ces analyses de médiation sont présentés dans la figure 4 .

Effets indirects $1 a^{\prime}, 2 a^{\prime}$ et $3 a^{\prime}$

Les résultats montrent qu'une partie de l'effet de la TR sur la ST est médiatisé par la $\mathrm{LD}$ (indirect 1a'), $\beta=-.12, S E=.02,95 \%$ IC $=[-.17 ;-.08]$.

En revanche, l'effet de la TR sur le PSS n'est pas médiatisé par la LD., $\beta=-.06, t$ $(434)=-1.34, p=.18$. Cela implique que l'effet de médiation en série (indirect 2a') n'est pas pertinent. Cela va à l'encontre de notre hypothèse $4 \mathrm{a}$. Néanmoins, ces résultats appuient notre 
hypothèse générale qui stipule que les liens entre la TR et la ST ne seront pas de même nature (direct vs. indirect) selon que l'on utilise l'ERI ou le JDC-S pour mesurer le stress. C'est ce que ces résultats tendent à montrer.

Nous observons également qu'une partie de l'effet de la TR sur la ST est médiatisée par le PSS (indirect 3a'), $\beta=-.06, S E=.02,95 \% I C=[-.11 ;-.03]$.

\section{Comparaison des deux effets indirects significatifs}

Nous avons comparé, à l'aide de contrastes, l'effet indirect 1a' (médiation simple par LD) à l'effet indirect 3a' (médiation simple par PSS). Ces deux effets expliquent une part comparable de l'effet total de la TR sur la ST (respectivement $25 \%$ et $14 \%$ ). Pour comprendre comment la TR affecte la ST, il faut tenir compte d'une mesure de stress interactionniste et d'une mesure de stress subjectif dans un modèle de médiations parallèles. Les différences entre les figures 3 et 4 montrent que les relations entre les tensions de rôle et la satisfaction au travail ne sont pas de même nature selon le modèle interactionniste de stress choisi. En effet, nous observons des médiations en série avec l'ERI et des médiations en parallèle avec le JDCS. Cela appuie notre hypothèse $2 \mathrm{c}$.

\section{INSERER FIGURE 4}

\section{Tension de rôle TR (VI), ERI (VM1), PSS10 (VM2) et fatigue nerveuse FN (VD)}

Les résultats concernant ces analyses de médiations sont présentés dans la figure 5. Rappelons que plus la TR augmente, plus la FN augmente $(r=.4, p<.0001)$. Ce résultat appuie notre hypothèse $3 \mathrm{~b}$.

\section{Effets indirects $1 b, 2 b$ et $3 b$}

Nos données appuient l'idée selon laquelle une partie de l'effet de la TR sur la FN est médiatisé par l'ERI (indirect $1 \mathrm{~b}$ ), $\beta=.11, S E=.02,95 \%$ IC $=[.06 ; .16]$.

Les résultats appuient notre hypothèse $4 \mathrm{~b}$ selon laquelle la TR aurait un effet sur la FN via l'ERI et le PSS (indirect 2b), $\beta=.06, S E=.04,95 \% I C=[.04 ; .08]$. Autrement dit, l'effet de la TR sur la FN suit bien une médiation en série impliquant l'ERI et le PSS.

Nous observons également que l'effet de la TR sur la FN est en partie médiatisé par le PSS (indirect 3b), $\beta=.10, S E=.03,95 \%$ IC $=[.05 ; .16]$. 


\section{Comparaison des trois effets indirects}

Nos tests de contrastes indiquent que les trois effets indirects expliquent une part de l'effet total comparable (indirect $1 b=28 \%$; indirect $2 b=16 \%$; indirect $3 b=26 \%$ ). Il semble que ces trois médiations permettent de comprendre les effets de la TR sur la FN, ce qui est conforme à notre hypothèse $4 \mathrm{~b}$.

\section{INSERER FIGURE 5}

\section{Tension de rôle TR (VI), demande psychologique DP (VM1), PSS10 (VM2) et fatigue nerveuse $\mathrm{FN}$ (VD)}

Les résultats concernant ces analyses de médiations sont présentés dans la figure 6 .

Effets indirects $1 b^{\prime}, 2 b^{\prime}$ et $3 b^{\prime}$

L'effet de la TR sur la FN est médiatisé par la DP (indirect 1b'), $\beta=.27, S E=.06,95 \%$ $I C=[.06 ; .15]$.

Conformément à notre hypothèse 4, nos résultats appuient l'idée d'une médiation en série (indirect 2b'). Cela signifie que la TE a un effet sur la FN en passant par l'intermédiaire de la DP qui passe elle-même par le PSS, $\beta=.05, S E=.03,95 \% I C=[.03 ; .07]$. Bien que cet effet soit faible ce résultat appuie notre hypothèse $4 \mathrm{~b}$.

Nous observons également que qu'une partie de l'effet de la TR sur la FN passe uniquement par le PSS (indirect 3b'), $\beta=.12, S E=.03,95 \%$ IC $=[.07 ; .17]$.

\section{Comparaison des trois effets indirects}

L'effet indirect 1b' (médiation simple par la DP) explique 26\% de l'effet total de la TR sur la FN, l'indirect 2b' (médiation en série) explique 13\% et l'indirect 3b' (médiation simple par PSS) explique 30\%. Même si l'indirect 2b' explique une part moins importante de l'effet total, il ajoute un apport non négligeable dans la compréhension des effets de la TR sur la FN. Ces résultats appuient notre hypothèse $4 \mathrm{~b}$.

Les médiations en série observées avec l'ERI (cf. figure 5) sont plus fortes que celles observées avec le JDC-S (cf., figure 6). Cela appuie notre hypothèse 2c. En effet, les effets indirects n'ont pas le même poids en fonction du modèle de stress choisi (ERI vs. JDC-S).

\section{INSERER FIGURE 6}




\section{Discussion}

\section{a. Des niveaux de prévalence du stress différents}

Selon le cadre d'analyse, JDC-S, ERI ou PSS, le taux de population en situation de RPS lié au stress est différent : respectivement $23.8 \%, 14.18 \%$ ou $42.33 \%$. Les rapports aux normes diffèrent également : la prévalence obtenue par le JCQ est inférieure à la norme, alors que celles issues de l'ERI ou du PSS positionnent notre population au-delà des résultats moyens nationaux. Quel chiffre reflète vraiment la réalité de l'entreprise ? Chaque outil d'analyse renvoie bien à une conception du stress au travail spécifique et mesure des dimensions différentes et complémentaires. Ces résultats confirment le rôle déterminant de la conception du stress au travail, donc du modèle théorique, dans son évaluation. Le choix du modèle n'est pas neutre pour l'intervention. En effet, si les processus et les facteurs investigués diffèrent, cela peut amener à sur ou sous-estimer certaines dimensions en jeu et les leviers d'action proposés à l'entreprise seront nécessairement eux aussi différents.

\section{b. La force des liens entre les modèles de stress et la santé psychologique au travail diffère}

Les liens entre les différents modèles de stress et la SPT montrent des différences. Le PSS est le modèle qui corrèle le plus fortement avec la fatigue nerveuse tandis que le JDC-S (par l'intermédiaire de la latitude décisionnelle) corrèle le plus fortement avec la satisfaction. Par ailleurs, les tensions de rôles (le facteur de stress que nous présentons ici), entretiennent des liens plus forts avec l'ERI, la demande psychologique et le soutien social qu'avec le PSS (cf. tableau 2).

Connaître les facteurs les plus liés à un modèle ou l'autre (JDC-S vs. ERI vs. PSS), ainsi que les conséquences du stress les plus corrélées à chaque facteur est important. En effet, les résultats attendus d'une intervention (agir sur la satisfaction ou sur la fatigue nerveuse) peuvent dépendre de la sensibilité des outils. Or certains (JDC-S) semblent plus adaptés pour étudier le versant positif de la SPT (satisfaction) et d'autres (PSS et ERI) pour étudier le versant négatif (fatigue nerveuse). Le choix d'un modèle peut également entraîner une présélection implicite des facteurs explicatifs du stress, qui aura une influence capitale sur les interventions de terrain qui vont en résulter. Nous soutenons, comme Althaus, Kop et 
Grosjean (2014), qu'il est nécessaire d'éclaircir les implicites relatifs aux choix des variables intégrées ou non dans les différents modèles et de l'impact que cela peut avoir sur les phénomènes décrits pour intervenir sur la santé au travail. «Le recours à un modèle théorique dont les partis pris sont explicités cadre l'action en l'orientant sciemment sur certaines catégories de variables pertinentes. ... les publications ne répondent guère à ces questions » (p.17). Nous soulignons d'ailleurs que cette recommandation peut être généralisée à d'autres travaux hors des champs du stress ou de la SPT.

\section{c. Une coexistence d'éléments positifs et négatifs}

Déjà Selye en 1956 considérait que l'on devait tenir compte simultanément du distress (détresse) et de l'eustress (bien-être) pour bien évaluer les tensions psychologiques vécues par une personne (Savoie et al., 2010). Comme le soulignent Gilbert et al. (2011), la SPT doit être considérée non comme un continuum allant de la maladie à l'absence de maladie (santé) mais comme un construit bidimensionnel, composé d'éléments positifs et négatifs. Dans notre échantillon, comprendre pourquoi $23.8 \%$ des sujets sont en jobstrain et isostrain se justifie bien sûr par rapport aux conséquences dramatiques du stress mais cela ne doit pas nous priver des pistes d'action que peuvent représenter les facteurs qui font que $76.2 \%$ des salariés ne sont pas en situation de risque.

Nos résultats vont dans le sens de cette bi-dimensionnalité, avec la présence simultanée d'éléments positifs (satisfaction) et négatifs (fatigue nerveuse). Les tensions de rôle semblent influencer les deux versants de la SPT mais de manière différente. Pour le versant positif, nous avons montré que la médiation en série n'est pas (ou peu) nécessaire pour comprendre l'impact des tensions de rôle sur la satisfaction. Sur le versant négatif, la médiation en série explique une part conséquente des effets des tensions de rôle sur la fatigue nerveuse. Plus concrètement, ces résultats montrent que le stress perçu est lié au stress mesuré par un modèle interactionniste quand on étudie les éléments négatifs du vécu expérientiel au travail (fatigue nerveuse), mais ce n'est pas le cas pour les éléments positifs. Autrement dit, ces résultats appuient l'idée selon laquelle la satisfaction au travail n'est pas en premier lieu l'absence de perception subjective de stress.

\section{d. La subjectivité des acteurs}

Dans quasiment tous les cas, tenir compte du stress perçu semble nécessaire pour améliorer la compréhension des phénomènes que nous cherchons à expliquer. En effet, que ce 
soit dans des médiations en série ou dans des médiations en parallèle, le stress perçu augmente la part de variance expliquée de la SPT. Certes, le lien entre la latitude décisionnelle et le stress perçu $(\beta=-.06)$ s'avère non significatif mais cette absence d'effet est intéressante. Elle tend à montrer que la latitude décisionnelle n'a pas toujours l'effet protecteur que suggère le modèle JDC-S. De plus, ce résultat appuie les travaux qui montrent qu'une trop grande latitude décisionnelle peut être néfaste pour la santé mentale (Legault \& BelarbiBasbous, 2006 ; Niedhammer et al., 2006). L'impact réel d'interventions primaires ne pourra être compris et être efficace qu'en intégrant la subjectivité des sujets. Augmenter la latitude décisionnelle ou diminuer la charge de travail, préconisations résultant souvent d'une intervention s'appuyant sur le modèle JDC-S, n'auront pas un effet mécanique et peuvent même potentiellement ne pas améliorer le stress perçu voire l'augmenter si une grande autonomie est perçue par le sujet comme le mettant en difficulté.

\section{Limites et conclusion}

Des limites peuvent être identifiées dans la réalisation de cette recherche. Tout d'abord, le taux très faible (38.8\%) de questionnaires valides pose question. L'échantillon de participants n'est probablement pas parfaitement représentatif de l'ensemble des salariés de l'organisation en question. De plus, nous ne pouvons pas exclure qu'un biais de désirabilité sociale ait affecté les réponses des participants. Enfin, nous n'avons pas pu montrer dans la présente recherche comment différents facteurs de stress au travail (faible justice organisationnelle, pression temporelle, pression à la productivité...) pouvaient influer différemment sur des mesures de stress et de SPT. Nous comptons explorer cette voie dans des travaux futurs en comblant les limites de cette recherche.

Sur le plan de l'intervention comme sur celui de la recherche fondamentale, plusieurs conclusions peuvent être tirées de cette recherche.

Tout d'abord, il semble nécessaire d'intégrer plus de variables aux travaux sur la santé psychologique au travail que ne le font les modèles classiques du stress. Un modèle est par définition une simplification de la réalité, mais cela ne dispense pas de s'interroger sur les conséquences du choix de ce modèle. Dans quelle mesure et de quelle façon le choix du modèle conditionne-t-il d'autres choix impactant la façon dont on va aborder le phénomène étudié ? On a vu dans cette recherche que le modèle de stress conditionne très largement le type et l'intensité des liens avec les variables indépendantes et dépendantes étudiées. Il faut donc trianguler les analyses en croisant plusieurs modèles et en leur adjoignant d'autres 
mesures intégrant d'autres facteurs de stress. De même, il semble pertinent d'adjoindre des mesures des conséquences du stress afin de dépasser une approche strictement épidémiologique.

Cette recherche nous amène également à nous interroger sur le choix du paradigme dans lequel un(e) psychologue va se situer et sur l'intérêt de passer d'un paradigme RPS à un paradigme QVT. Différents éléments distinguent ces deux paradigmes, comme nous l'avons déjà argumenté :

- la vision du travail: facteur de risque, source de souffrance ou facteur de développement et de bien-être, source d'identité et de plaisir ;

- la définition de la santé psychologique au travail : continuum unidimensionnel (être en bonne santé c'est ne pas être en mauvaise santé) ou combinaison de deux dimensions interdépendantes (détresse et bien être psychologique au travail);

- la vision du sujet : victime passive ou acteur, c'est à dire capable d'actions et dont il faut considérer toute la subjectivité, tenir compte de ses interprétations et de la façon dont il va en retour construire ou reconstruire, à titre individuel et collectif (Loriol, 2016), à la fois ses représentations et son environnement ;

- au final la vision de l'organisation elle-même : «coupable» de la souffrance de ses salariés ou co-acteur, co-porteur avec le sujet de la responsabilité de la santé au travail (Chakor, 2011).

$\mathrm{Au}$ vu de nos résultats, qui soulignent notamment l'importance de prendre en compte la bi-dimensionnalité de la SPT et la subjectivité des acteurs, le paradigme QVT paraît plus adapté. À la condition toutefois que ce paradigme QVT intègre à la fois les éléments positifs et négatifs du vécu expérientiel au travail. Sinon ce ne sera qu'un passage de travaux principalement centrés sur les seules dimensions négatives du travail à des travaux principalement centrés sur les aspects positifs de la santé psychologique au travail.

\section{Références}

Allard-Poesi, F., \& Hollet-Haudebert, S. (2012). La construction du sujet souffrant au travail au travers des instruments scientifiques de mesure. @ GRH, (4), 45-74. 
Althaus A., Kop, JL, \& Grosjean, V. (2013). Recension critique des modèles théoriques reliant environnement de travail, stress et sante : vers un meta-modèle. Le travail humain, $76(2), 81-103$

Burakova, M., \& Leduc, S. (2014). Risques psychosociaux, Qualité de vie au travail: Opposition ou complémentarité? De la prévention à la promotion de la santé au travail. Santé au Travail et Risques Psychosociaux: Tous Préventeurs, 73-96.

Cammann, C., Fichman, M., Jenkins, G.D., Jenkins, G.D., Jr., \& Klesh, J.R. (1983). Assessing the attitudes and perceptions of organizational members. In S.E. Seashore, E.E. Lawler III, P.H. Mirvis \& C. Cammann (Eds.), Assessing organizational change: A guide to methods, measures, and practices (pp. 71 - 138). New York, NY : Wiley.

Chakor, T. (2011). Le consultant dans la prévention des risques psychosociaux: des positionnements éthiques différenciés. HAL

Clot, Y. (2015). Le travail à cour: pour en finir avec les risques psychosociaux. La découverte.

Codo, S. (2011), Inégalités des managers publics territoriaux face au stress professionnel : impact des tensions de rôle, Actes du 22ème Congrès de l'AGRH.

Cohen, S., Tyrrell, D. A., \& Smith, A. P. (1993). Negative life events, perceived stress, negative affect, and susceptibility to the common cold. Journal of personality and social psychology, 64(1), 131.

Crozier, M., \& Friedberg, E. (1977). L'acteur et le système. Paris: Editions du Seuil.

Dejours, C. (1993). Travail: usure mentale. De la psychopathologie à la psychodynamique du travail. Bayard, Paris.

Dejours, C. (2000). Travail. Usure mentale (3e édition). Paris: Bayard Éditions.

Elovainio, M., Kivimäki, M., Linna, A., Brockner, J., Van Den Bos, K., Greenberg, J., \& Vahtera, J. (2010). Does organisational justice protect from sickness absence following a major life event? A Finnish public sector study. Journal of Epidemiology \& Community Health, 64(5), 470-472.

Gilbert, M. H., Dagenais-Desmarais, V., \& Savoie, A. (2011). Validation d'une mesure de santé psychologique au travail. Revue européenne de psychologie appliquée/European Review of Applied Psychology, 61(4), 195-203.

Gollac, M., \& Bodier, M. (2011). Mesurer les facteurs psychosociaux de risque au travail pour les maîtriser. Rapport du Collège d'expertise sur le suivi des risques psychosociaux au travail. 
Grosjean, V., \& Guyot, S. (2016). Bien être et qualité de de vie au travail, risques psychosociaux: de quoi parle-t-on ?.Revue technique de l'INRS Hygiène et Sécurité au travail (242)

Guillet, L., Hermand, D., Py, Y. (2003). Stress et contraintes de travail : Un modèle cognitif exploratoire. In N. Delobbe, G. Karnas, C. Vandenberghe (Eds). Bien-être au travail et transformation des organisations. Presses Universitaires de Louvain, tome 3 p. 59-68.

Hayes, A. F. (2013). An introduction to mediation, moderation, and conditional process analysis: A regression-based approach. New York, NY: Guilford Press.

Karasek, R.A. \& Theorell, T. (1990). Healthy work: stress, productivity, and the reconstruction of working life. New York: Basic Books.

Karnas, G., \& Hellemans, C. (2002). Approche psycho-organisationnelle du stress professionnel: les limites d'un modèle. Stress au travail et santé psychique, 175-188.

Katz, D., \& Khan, R. L. (1978). Psicologia social das organizações. 2’ ed. São Paulo: Atlas.

Laberon, S., Barou, D., \& Ripon, A. (2016). Management et risques psychosociaux dans l'institution militaire risques psychosociaux au sein de l'institution militaire: l'effet médiateur du style de management perçu. Le travail humain, 79(4), 363-393.

Lazarus, R. S., \& Folkman, S. (1984). Stress. Appraisal and Coping. New York: Springer.

Loriol, M. (2010). Agir contre le stress et les risques psychosociaux au travail. Regards sur l'actualité: mensuel de la vie publique en France, (363), 52-63.

Loriol, M. (2016). Les dimensions collectives de la qualité de vie au travail. La Revue des Conditions de Travail, (3), 25-32.

Le Blanc, P., de Jonge, J., \& Schaufeli, W. B. (2000). Job stress and health. Blackwell Publishing.

Legault, M. J., \& Belarbi-Basbous, H. (2006). Gestion par projets et santé mentale au travail dans la nouvelle économie. Pistes, 8(1).

Niedhammer, I., Siegrist, J., Landre, M. F., Goldberg, M., \& Leclerc, A. (2000). Étude des qualités psychométriques de la version française du modèle du Déséquilibre Efforts/Récompenses. Revue d'épidémiologie et de santé publique, 48(5), 419-438.

Niedhammer, I., Chastang J.F., Gendrey, L., David, S., \& Degioanni, S. (2006). Propriétés psychométriques de la version française des échelles de la demande psychologique, de la latitude décisionnelle et du soutien social du «Job Content Questionnaire» de Karasek: résultats de l'enquête nationale SUMER. Santé publique, 18(3), 413-427. 
Rascle, N., \& Irachabal, S. (2001). Médiateurs et modérateurs: implications théoriques et méthodologiques dans le domaine du stress et de la psychologie de la santé. Le travail humain, 64(2), 97-118.

Ribert-Van de Weerdt, C. (2011). Les contraintes de travail et les stratégies de régulation émotionnelle en centre de relation clientèle. Le travail humain, 74(4), 321-339.

Rizzo J R, House R J, Lirtzman S I (1970), Role conflict and ambiguity in complex organizations, Administrative Science Quaterly, 15(1), 150-63

Ruiller, C. (2008), Le soutien social au travail : conceptualisation, mesure et influence sur l'épuisement professionnel et l'implication organisationnelle : l'étude d'un cas hospitalier, Thèse de doctorat en sciences de gestion, Université Rennes 1.

Savoie, A., Brunet, L., Boudrias, J. S., \& Gilbert, M. H. (2010). Surenchère de la non-santé psychologique au travail. Le journal des psychologues, (10), 31-34.

Seligman, M. E., \& Csikszentmihalyi, M. (2000). Special issue on happiness, excellence, and optimal human functioning. American Psychologist, 55(1), 5-183.

Selye, H. (1956). The stress of life.

Siegrist, J. (1991). Contributions of sociology to the prediction of heart disease and their implications for public health. The European Journal of Public Health, 1(1), 10-21.

Van Wassenhove., W., (2014). Modèle de Karasek. In Zawieja, P., Guarnieri, F. (Eds). Dictionnaire des risques psychosociaux. Paris: Le Seuil, pp.170-174

Veit, C. T., \& Ware, J. E. (1983). The structure of psychological distress and well-being in general populations. Journal of consulting and clinical psychology, 51(5), 730.

Xie, D. (2007), Buffering or strengthening: the moderating effect of self-efficacy on stressorstrain relationship, Journal of Career Assessment, vol. 15, n 3, p. 351-366. 

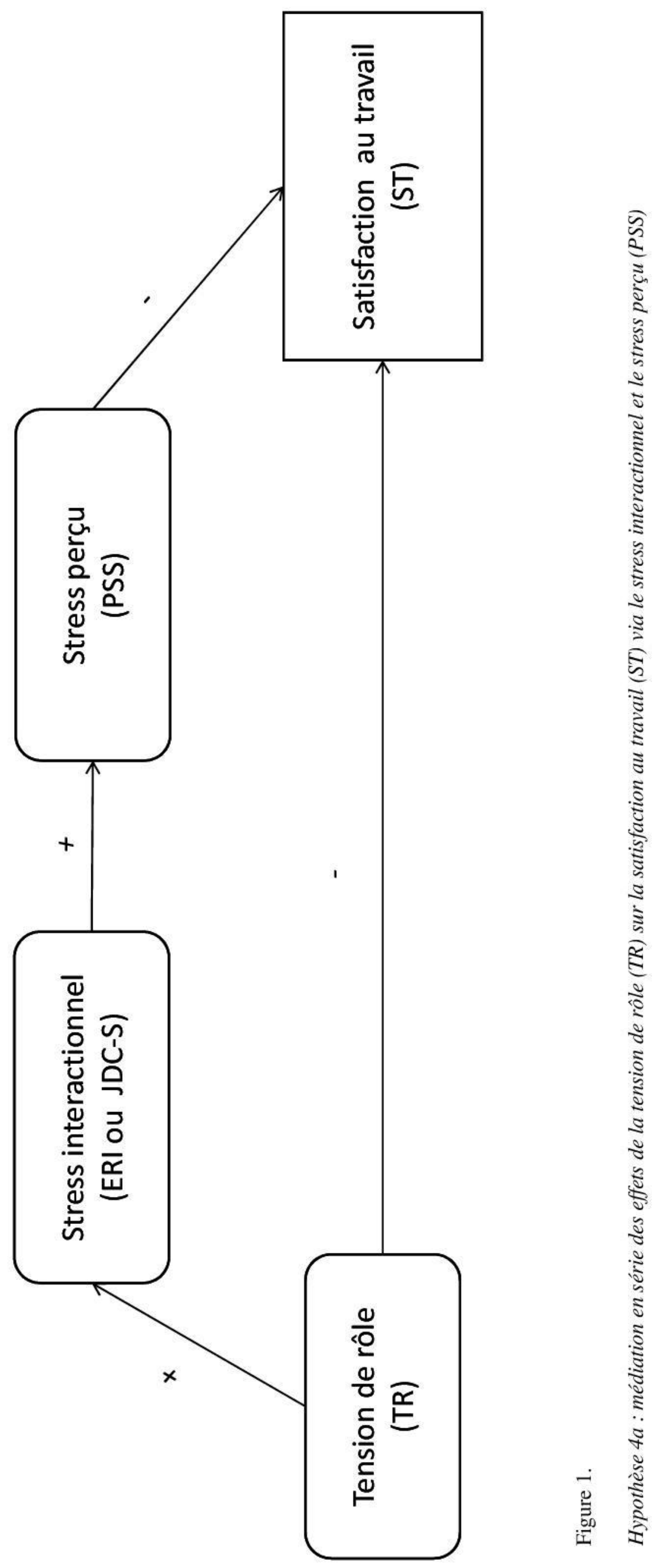


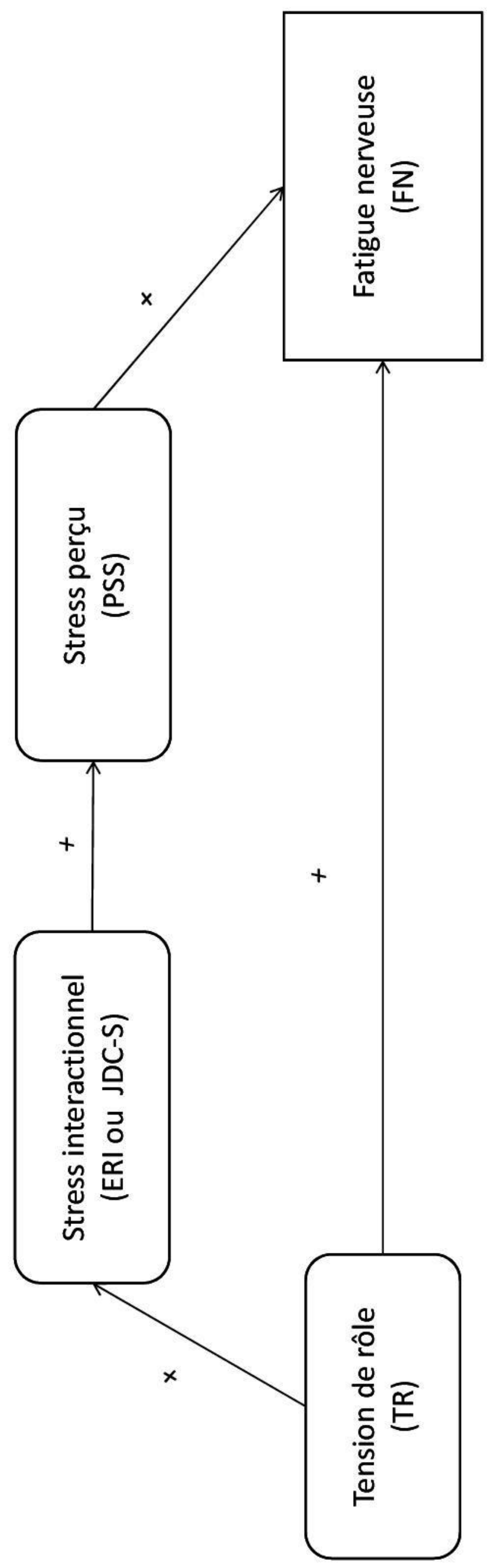

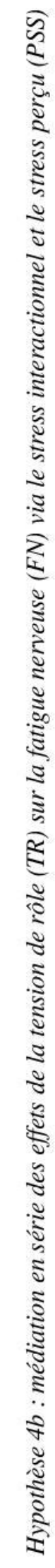



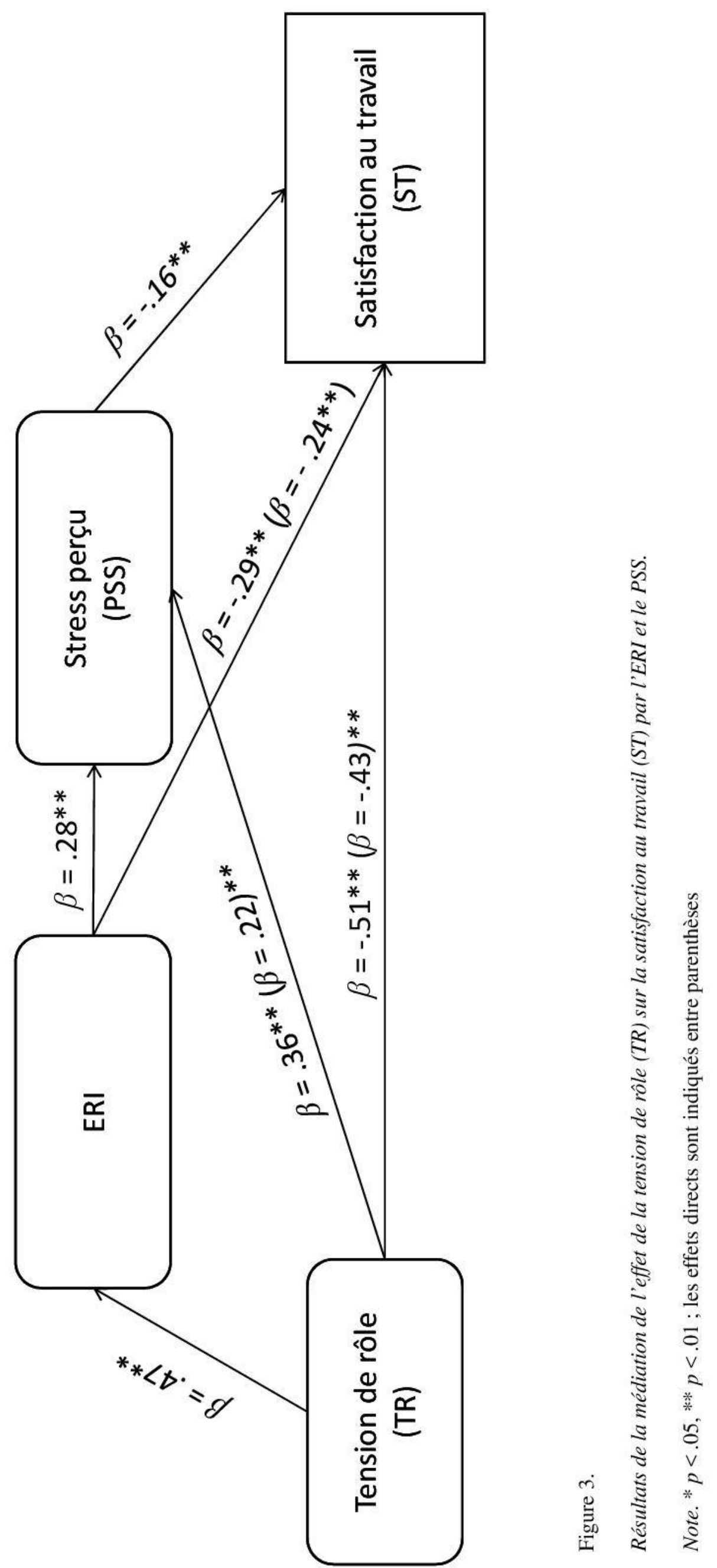


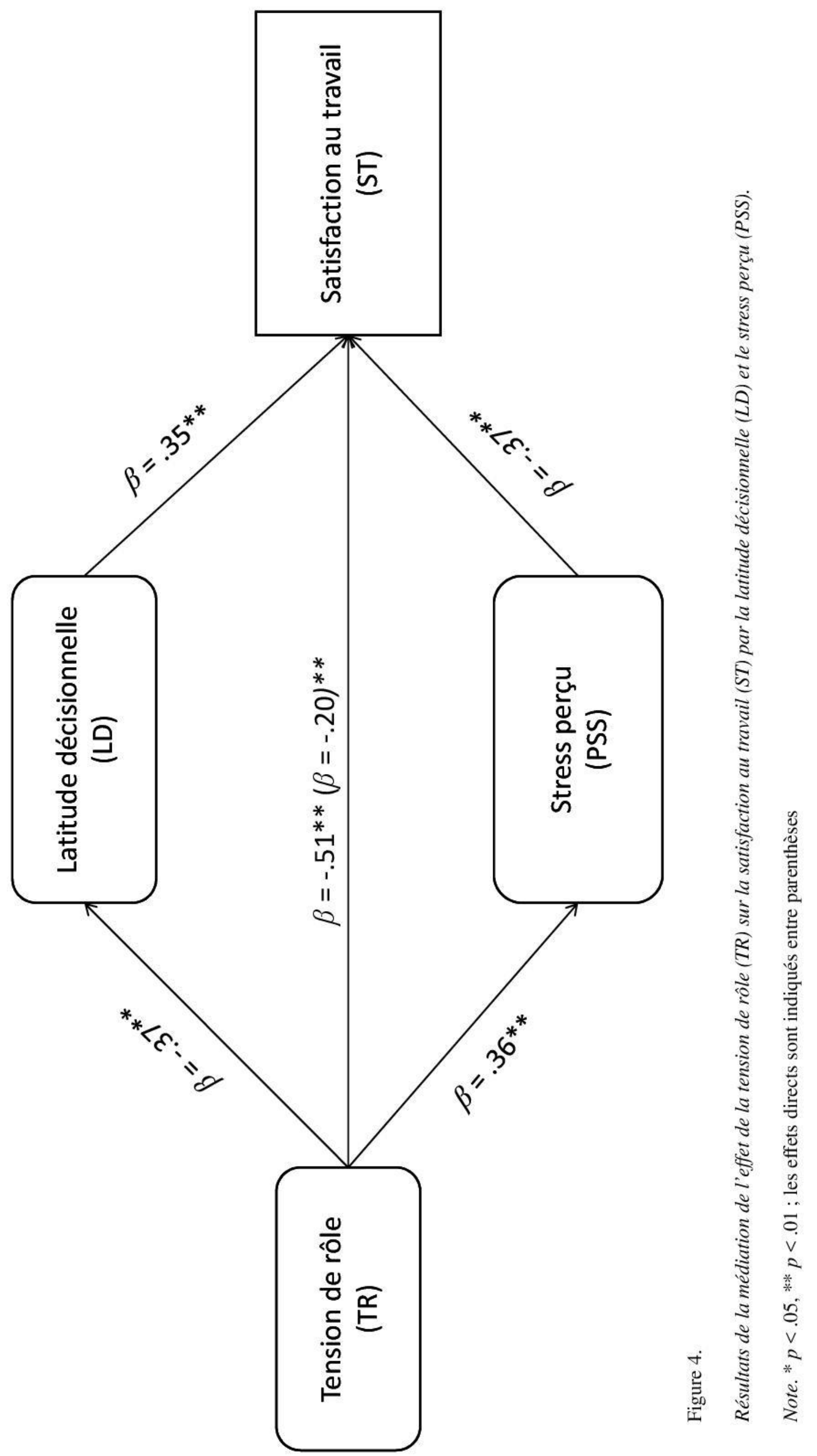



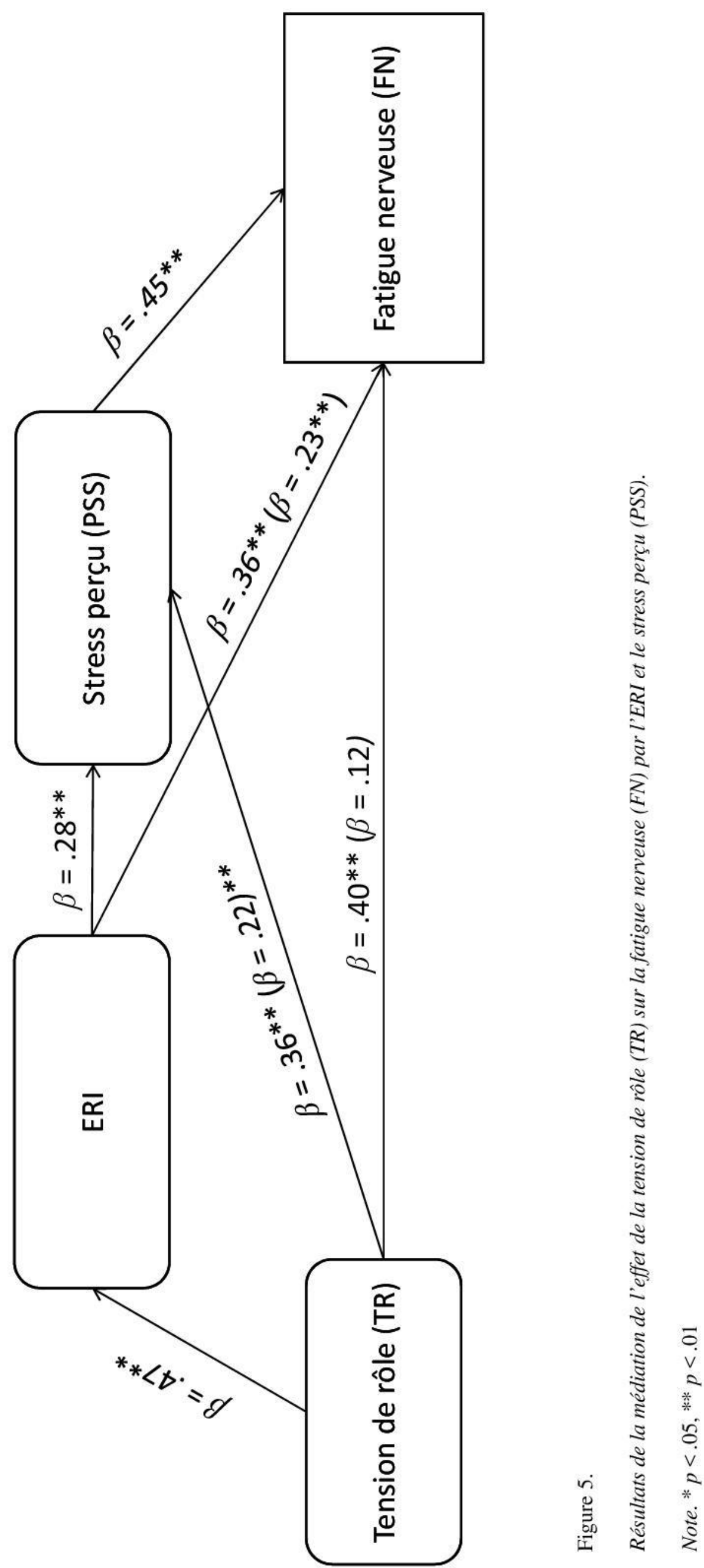

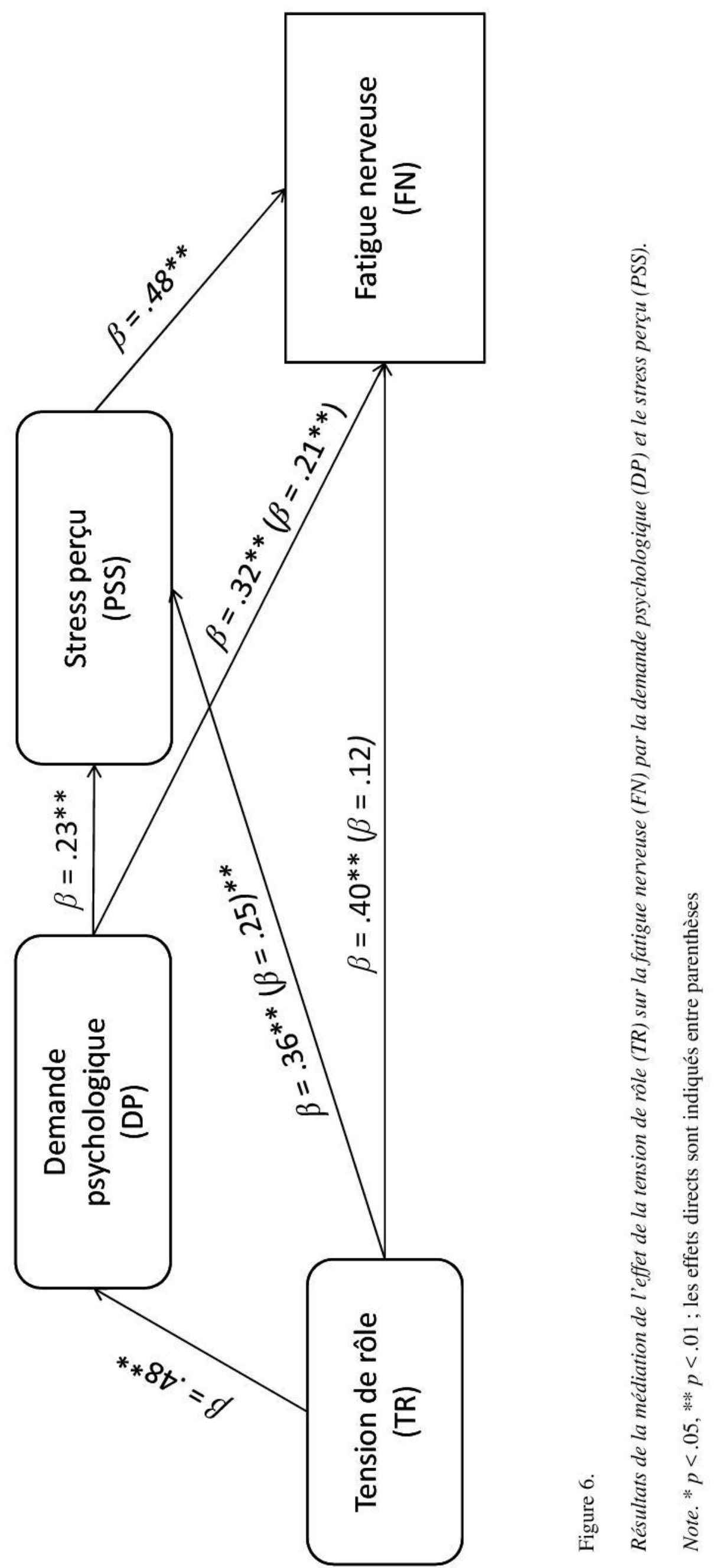


\begin{tabular}{|l|l|l|}
\hline Modèle & Résultats & Normes de référence \\
\hline JDC-S (Karasek) & $\begin{array}{l}\text { Jobstrain }: 4.8 \% \\
\text { Isostrain }: 19 \% \\
\text { Total : } 23.8 \%\end{array}$ & $\begin{array}{l}\text { Jobstrain }: 23.2 \% \\
\text { Isostrain }: 14.5 \% \\
\text { Total }: 37.7 \% \\
\text { (Niedhammer et al. 2006) }\end{array}$ \\
\hline $\begin{array}{l}\text { ERI (Siegrist) }: \% \text { de sujets en } \\
\text { déséquilibre efforts / récompenses }\end{array}$ & $14.8 \%$ & $\begin{array}{l}6 \% \\
\text { (Niedhammer et al. 2000) }\end{array}$ \\
\hline $\begin{array}{l}\text { PSS (Cohen) }: \% \text { de sujets en sur-stress } \\
\text { (score }>27)\end{array}$ & $42.33 \%$ & $\begin{array}{l}23.8 \% \\
\text { (Poirot, 2004) }\end{array}$ \\
\hline
\end{tabular}

Tableau 1 : Prévalence du stress en fonction des différents modèles d'analyse 


\begin{tabular}{|c|c|c|c|c|c|c|c|c|c|}
\hline & Moyennes & SD & 1 & 2 & 3 & 4 & 5 & 6 & 7 \\
\hline 1. Tension de rôle (TR) & 2.19 & 0.49 & - & & & & & & \\
\hline 2. ERI ratio & 0.71 & 0.26 & $.47^{* *}$ & - & & & & & \\
\hline 3. Latitude décisionnelle (LD) & 63.19 & 15.81 & $-.37 * *$ & $-.32 * *$ & - & & & & \\
\hline 4. Demande psychologique (DP) & 22.24 & 4.80 & $.48^{* *}$ & $.45^{* *}$ & -.07 & - & & & \\
\hline 5. Soutien social (SoS) & 22.17 & 4.20 & $-.54 * *$ & $-.46 * *$ & $.49 * * *$ & $-.33 * * *$ & - & & \\
\hline 6. Stress perçu (PSS) & 2.55 & 6.47 & $.36^{* *}$ & $.40 * *$ & $-.19 * *$ & $.35 * * *$ & $-.34 * *$ & - & \\
\hline 7. Satisfaction au travail (ST) & 3.14 & 0.6 & $-.48 * *$ & $-.45 * *$ & $.48 * * *$ & $-.31 * * *$ & $.48^{* *}$ & $\begin{array}{l}- \\
.37^{* *}\end{array}$ & - \\
\hline 8. Fatigue nerveuse & 2.18 & 0.91 & $.4 * *$ & $.47^{* *}$ & $-.13 * *$ & $.44 * * *$ & $-.38 * *$ & $.59 * *$ & $-.40 * *$ \\
\hline
\end{tabular}

Tableau 2 : Moyennes, écarts-types et corrélations entre les variables $N=436 ;{ }^{*} p<.05 ; * * p<.01$. 